\title{
PREDICCIÓN DEL NÚMERO DE PAROS DE PRODUCCIÓN EN LIÍNEAS DE ENSAMBLE
}

\author{
Prediction of the number of production \\ stoppages in assembly lines
}

Dra. Gema Karina Ibarra Torúa ${ }^{1}$

M.I. David Ruiz Aguirre ${ }^{2}$

Dr. Dagoberto Burgos Flores 3

Ilse Irene Yepis Osuna 4

EPISTEMUS

ISSN: 2007-8196 (electrónico)

ISSN: 2007-4530 (impresa)

Gilberto Orrantia-Daniel ${ }^{1}$

Jaime Sánchez-Leal ${ }^{2}$

Jorge de la Riva-Rodríguez ${ }^{3}$

Manuel Rodríguez-Medina 4

Rosa María Reyes-Martínez ${ }^{5}$

Recibido: 12 de septiembre de 2016,

Aceptado: 30 de noviembre del 2016

Autor de Correspondencia:

M.C. Gilberto Orrantia-Daniel

Correo: orrantiadaniel@hotmail.com

\section{Resumen}

Es presentada una metodología para evaluar la condición actual y predecir la causa de inactividad de líneas de ensamble realizando un análisis del número de paros de producción. Es investigado el comportamiento de los paros por causa y por tipo de estación de trabajo, para así orientar a una mejor toma de decisiones. Los datos recolectados fueron la estación que provoca el paro de línea, su causa y el número de paros. Los cálculos obtenidos fueron las probabilidades de las causas de paro, aplicando la prueba de bondad de ajuste chi-cuadrada. En base a la distribución multinomial, fueron presentados modelos para predecir las causas de los próximos " $m$ " paros de la línea. Además, uno de los principales hallazgos fue que los paros debidos a los operadores son el $61.37 \%$ de todos los paros, por lo que es recomendado maximizar las habilidades y conocimientos de los operadores.

Palabras clave: Líneas de ensamble, prueba chi-cuadrada, distribución multinomial, paro de línea.

\section{Abstract}

A methodology is presented to evaluate the current condition and predict the cause of inactivity of assembly lines by performing an analysis of the number of production stoppages. The behavior of the stoppages is investigated by cause and by type of work station, in order to guide better decision making. The data collected was the station that causes the line stoppage, its cause and the number of stoppages. The calculations obtained were the probabilities of the causes of stoppage, applying the chi-square goodness of fit test. Based on the multinomial distribution, models were presented to predict the causes of the next " $m$ " stoppages of the line. In addition, one of the main findings was that the stoppages due to operators are $61.37 \%$ of all stoppages, so it is recommended to maximize the skills and knowledge of the operators.

Keywords: Assembly lines, chi-square test, multinomial distribution, line stoppag

1 Tecnológico Nacional de México/l. T. Hermosillo, Departamento de Ingeniería Industrial. Hermosillo, Sonora, México. Correo: orrantiadaniel@hotmail.com

2 Tecnológico Nacional de México/l. T. Ciudad Juárez, División de Estudios de Posgrado e Investigación. Ciudad Juárez, Chihuahua, México. Correo: jsanchez@itcj.edu.m

3 Tecnológico Nacional de México/l. T. Ciudad Juárez, División de Estudios de Posgrado e Investigación. Ciudad Juárez, Chihuahua, México. Correo: jriva@itcj.edu.mx

4 Tecnológico Nacional de México/l. T. Ciudad Juárez, División de Estudios de Posgrado e Investigación. Ciudad Juárez, Chihuahua, México. Correo: manuel rodriguez itci@yahoo.com

5 Tecnológico Nacional de México/l. T. Ciudad Juárez, División de Estudios de Posgrado e Investigación. Ciudad Juárez, Chihuahua, México. Correo: rosyreyes2001@yahoo.com 


\section{INTRODUCCIÓN}

En los sistemas de producción existe la preocupación por tener procesos esbeltos y con ello reducir y eliminar los desperdicios [1]. Parte de la manufactura esbelta consiste en la búsqueda de la eliminación de desperdicios. El desperdicio es cualquier cosa que aumenta el costo, pero no el valor a un producto. Uno de los siete desperdicios es la espera, reflejada en retrasos, paros y en procesos u operaciones detenidas esperando al proceso anterior [2].

Los paros inesperados y constantes en las estaciones de una línea de ensamble pueden tener consecuencias en el funcionamiento general de la línea. El efecto negativo de los paros en líneas de ensamble con ritmo continuo no sólo afecta a la estación de trabajo que generó el paro, sino que también obliga a que todas las estaciones de trabajo dejen de funcionar, sin importar si están antes o después de la estación en paro, causando un vacío en la productividad de toda línea.

\section{OBJETIVO}

Es buscado evaluar la condición actual y predecir el número de paros de producción según la causa de inactividad en líneas de ensamble por medio de un análisis del número de paros de línea. El objetivo es interpretar el comportamiento de los paros por causa y por tipo de estación de trabajo, para así orientar a una mejor toma de decisiones a los administradores de las líneas de ensamble. Los datos recolectados fueron la estación que provoca el paro de línea, su causa y el número de paros.

\section{MARCO TEÓRICO Y TRABAJO PREVIO}

El tiempo de paro es la interrupción de alguna operación por descomposturas de máquinas o herramientas, falta de material, entre otros [3]. A partir de la anterior definición, es determinado que un paro de línea es una interrupción de una operación realizada en alguna estación de trabajo.

Una línea de ensamble a ritmo tiene un sistema de cinta transportadora que mueve los puestos de trabajo (por ejemplo, automóviles) desde una estación de trabajo a la siguiente, a una velocidad constante. Si el ensamble en una estación en particular es realizado manualmente, los trabajadores caminan al lado de la cinta transportadora mientras desempeñan su operación, al finalizar caminan de regreso al punto en la línea que corresponde al comienzo de su estación o sección [4].

Existen estudios que analizaron los paros de producción, cuyo objetivo fue una identificación rápida del problema para eliminarlo como lo presenta Xi Gu et al. [5], sin embargo, fue detectado que son pocos los análisis de las causas que generan los paros de línea.

Uno de los estudios relacionado con el análisis de las causas de los paros de línea fue realizado por Panagiotis $\mathrm{H}$. Tsarouhas [6], quien desarrolló una metodología para clasificar los modos de falla principales en categorías para una línea de producción de pan durante un periodo de 2 años. Estimó las probabilidades de estas categorías aplicando la prueba de bondad de ajuste chi-cuadrada, y calculó las probabilidades conjuntas de la función de masa para estación de trabajo y a nivel de línea. Presentó ejemplos numéricos con el fin de predecir las causas y frecuencias de averías para estaciones de trabajo y para la línea de producción de pan que será producida en el futuro. La metodología está destinada como guía para fabricantes de productos de pastelería y panadería, mejorando el funcionamiento de las líneas de producción.

El estadístico Chi-cuadrada tiene aplicaciones en la verificación dehipótesis sobre una varianza yen los métodos de análisis de variables categóricas, generalmente con datos de variables continuas y categóricos específicamente

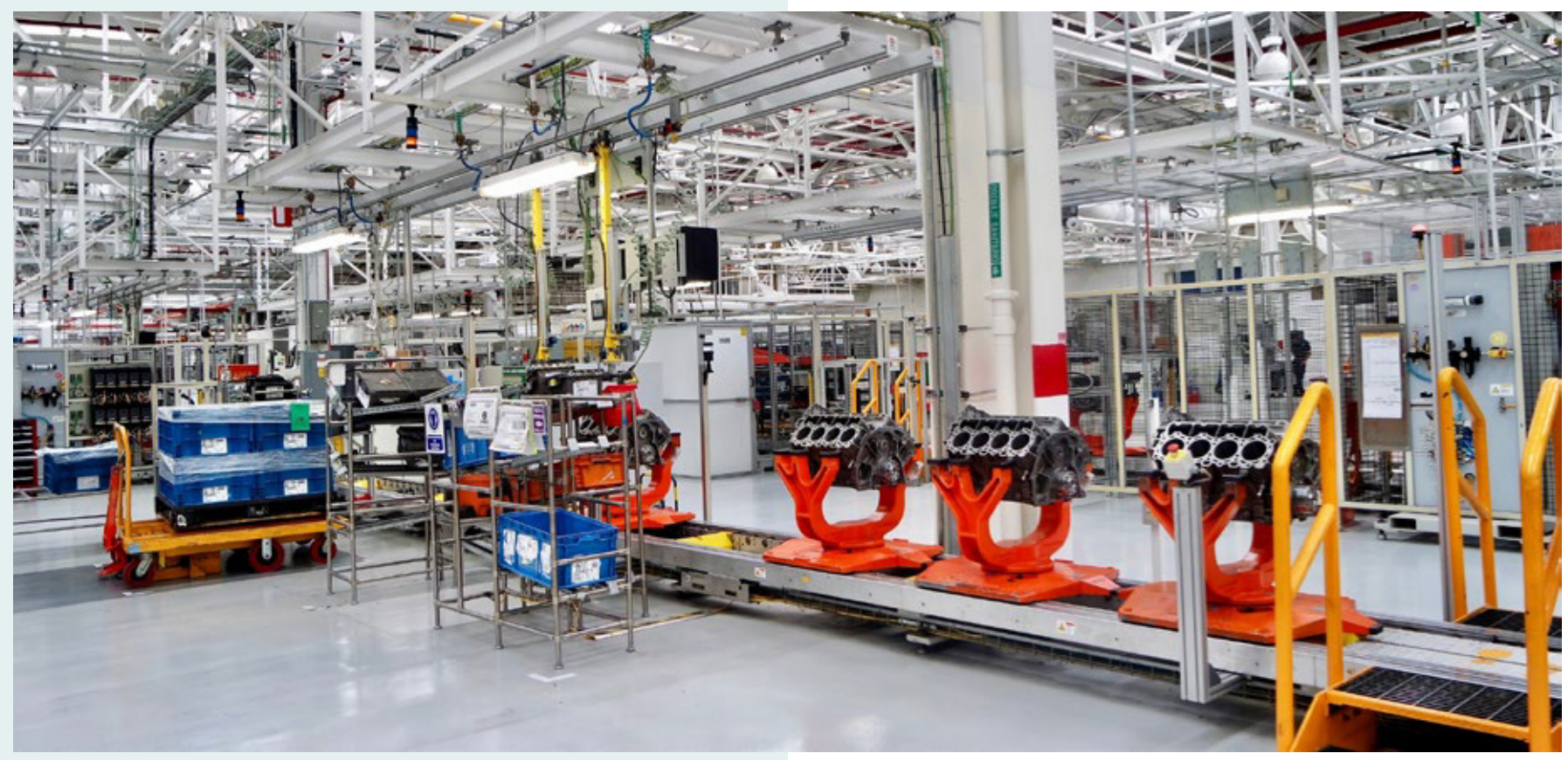


cuando se trata de datos binomiales, pero también es aplicable en datos categóricos en forma generalizada en función de variables multinomiales [7].

Al realizar un análisis de datos clasificados con dos o más niveles, la distribución binomial es apropiada para analizar las clasificaciones con dos niveles, mientras que la distribución multinomial es apropiada cuando hay tres o más niveles de clasificación. Las pruebas de hipótesis son referidas para evaluar si el vector de probabilidad de la distribución multinomial toma un valor especificado. La prueba de bondad de ajuste, a menudo expresada como prueba Chi-cuadrada, es utilizada para probar la hipótesis de homogeneidad (que establece que toda clasificación es igualmente probable) y la hipótesis de independencia. Este método también puede ser utilizado para probar las hipótesis de distribución de un conjunto de datos [8].

La prueba de bondad y ajuste verifica hipótesis sobre los valores de las proporciones los cuales provienen de teorías, leyes o aseveraciones, por ejemplo, si se desea comprobar la hipótesis de uniformidad, es decir que todas las categorías son igualmente probables, esto es que $P_{i}=1 / c$, para todos los valores. Básicamente la verificación consiste en ver si las observaciones se ajustan a los valores hipotéticos.

La prueba de bondad de ajuste está desarrollada por Jaime Sánchez [7], además están explicadas las pruebas de independencia y las pruebas de homogeneidad, las cuales serán utilizadas más adelante para el tratamiento de los datos. Además, por Jaime Sánchez [7] está explicado que la distribución multinomial es el modelo matemático de un experimento multinomial, cuyas observaciones pueden estar clasificadas en " $c$ " diferentes categorías o clases mutuamente excluyentes.

\section{PLANTEAMIENTO DEL PROBLEMA}

Los paros de producción de una línea de ensamble con ritmo continuo detienen la línea cada vez que al menos una

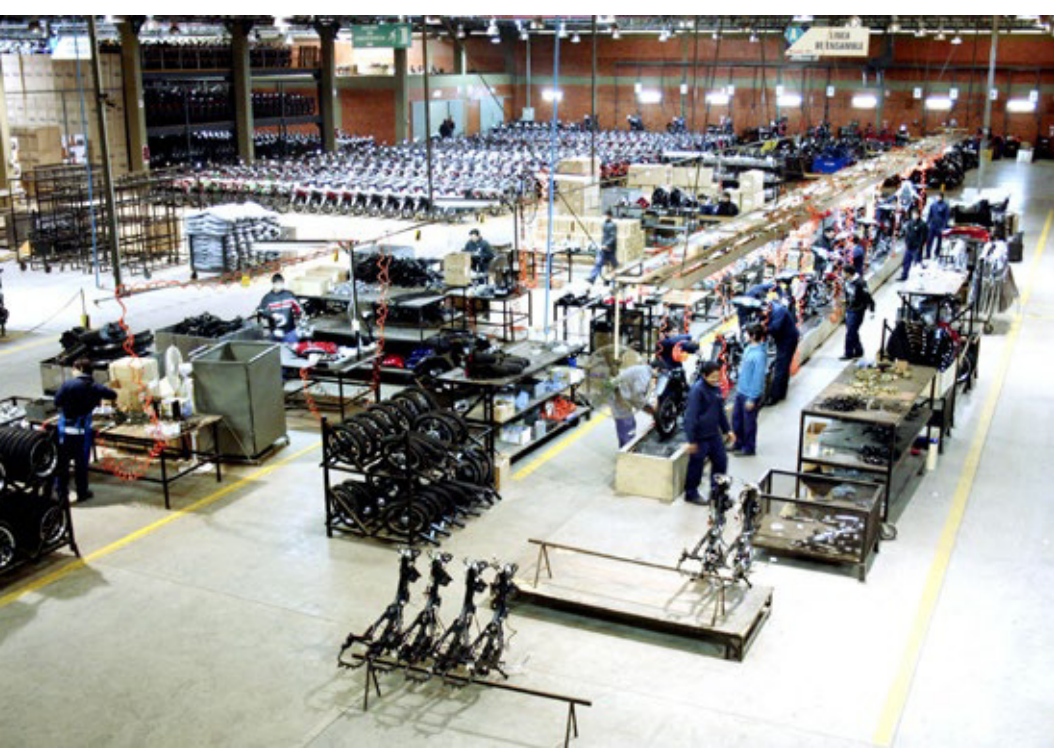

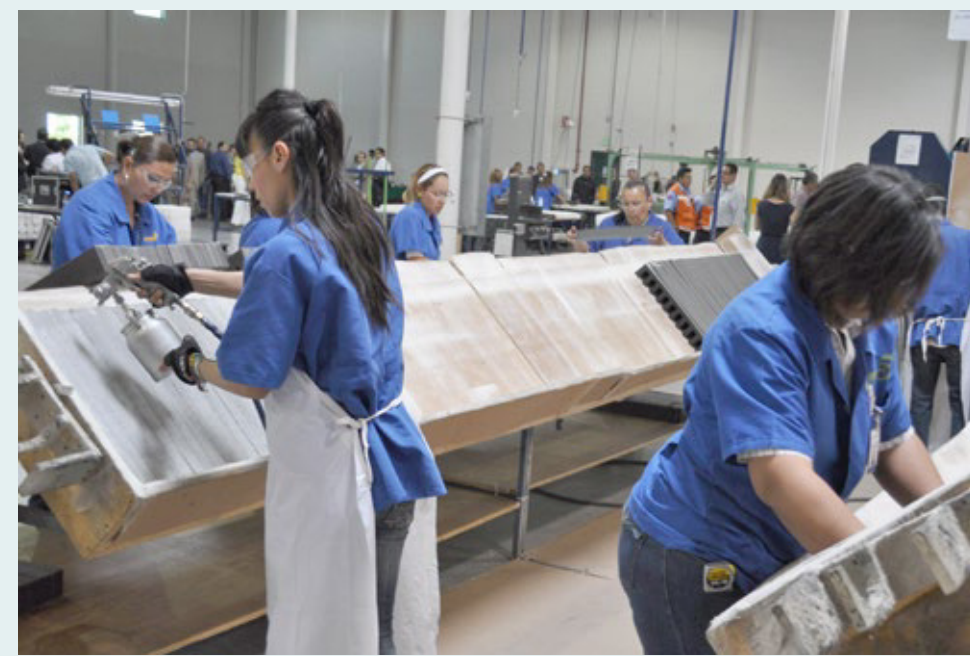

estación de trabajo requiere más tiempo que el asignado para culminar su tarea teniendo como consecuencia la falta de producción y el incumplimiento a las solicitudes de los clientes, entre otras. Esos paros son debidos a causas de diversas índoles que requieren ser evaluadas y que permita predecir los paros de línea, para así orientar la mejora de la administración de la operación de la línea de ensamble.

Este estudio está basado en lo realizado por Panagiotis H. Tsarouhas [6], solo que es aplicado en una línea de ensamble de la industria automotriz; la información es tomada de registros de mantenimiento y de la observación directa de la línea de ensamble; la duración de un paro en su mayoría es en segundos y a lo mucho en minutos. Además, detectamos que los problemas no solo los debía de atender el área de mantenimiento, si no que debían intervenir otras áreas en su solución.

La línea de ensamble que fue analizada está compuesta de varias estaciones de trabajo en serie. El movimiento de material entre estaciones es realizado automáticamente por un solo medio mecánico. Está formada por 34 estaciones de trabajo que en su mayoría realizan operaciones de ensamble manual con ayuda de herramientas manuales eléctricas (taladros) y esporádicamente son utilizados lectores manuales de códigos de barras (escáneres).

Cuando es producido un paro en alguna estación de trabajo, el primero en determinar lo que debe hacerse para eliminarlo, es el operador de la estación de trabajo y en segunda instancia el jefe de línea y el supervisor de producción. Ellos son los que evalúan la situación y dependiendo del problema solicitan intervención de los departamentos de soporte. Los principales departamentos de soporte son el de mantenimiento, ingeniería, calidad y materiales.

\section{MÉTODO DE TRABAJO}

Primeramente, fue recolectada una muestra de paros de línea para obtener un análisis inicial (ver tabla 1). El objetivo de ésta muestra fue agrupar las estaciones según la cantidad de paros de línea que generan. 
Tabla 1. Frecuencia de paros por estación de la línea de ensamble.

\begin{tabular}{|c|c|c|c|c|c|c|c|c|c|c|c|}
\hline Mue. & $\begin{array}{c}\text { Est. en } \\
\text { paro }\end{array}$ & Frec. & $\begin{array}{l}\text { Tipo de } \\
\text { estación }\end{array}$ & Mue. & $\begin{array}{c}\text { Est. en } \\
\text { paro }\end{array}$ & Frec. & $\begin{array}{l}\text { Tipo de } \\
\text { estación }\end{array}$ & Mue. & $\begin{array}{c}\text { Est. en } \\
\text { paro }\end{array}$ & Frec. & $\begin{array}{l}\text { Tipo de } \\
\text { estación }\end{array}$ \\
\hline 1 & 2 & 64 & B & 9 & 12 & 38 & B & 17 & 21 & 42 & $B$ \\
\hline 2 & 3 & 27 & C & 10 & 13 & 48 & B & 18 & 22 & 36 & B \\
\hline 3 & 4 & 30 & C & 11 & 14 & 86 & A & 19 & 23 & 80 & A \\
\hline 4 & 5 & 117 & A & 12 & 15 & 30 & $C$ & 20 & 24 & 38 & B \\
\hline 5 & 7 & 57 & B & 13 & 16 & 31 & C & 21 & 25 & 18 & C \\
\hline 6 & 8 & 30 & $C$ & 14 & 17 & 88 & A & 22 & 33 & 39 & B \\
\hline 7 & 10 & 197 & $A$ & 15 & 18 & 33 & $C$ & 23 & 34 & 56 & B \\
\hline 8 & 11 & 69 & B & 16 & 20 & 43 & B & & Total & 1297 & \\
\hline
\end{tabular}

Como es mostrado en la tabla 1 , fueron eliminadas aquellas estaciones con menos de 5 paros con el fin de cumplir con uno de los supuestos para realizar el análisis de medias que es presentado más adelante. Las estaciones eliminadas fueron la 1, 6, 9, 19, 26, 27, 28, 29, 30, 31, y 32 . El número total de paros inicialmente analizados fueron de 1297 paros de línea. Más adelante será explicado cómo se obtuvo la columna "tipo de estación".

Posteriormente fueron clasificadas las estaciones según la frecuencia de paros de línea con un análisis de medias para datos con distribución Poisson. Éste análisis de medias (también llamado ANOM) es equivalente al gráfico de ANOVA y a la prueba la igualdad de las medias de la población [9]. En nuestro caso, el gráfico ANOM fue utilizado para obtener tres grupos o tipos de estaciones; un grupo por arriba de la media, otro grupo alrededor de la media general y finalmente un grupo por debajo de la media, los cuales los identificamos con las letras " $A$ ", " $B$ " $y$ " $C$ " respectivamente (ver figura 1 y tabla 1 ).

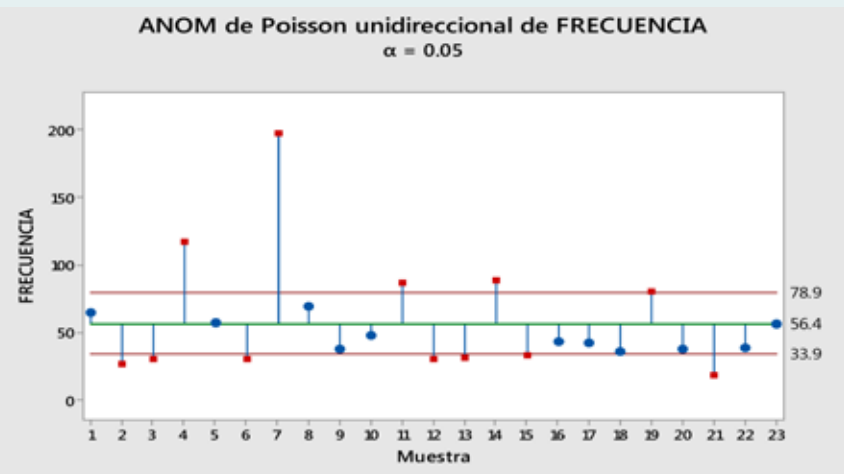

Figura 1. Gráfica ANOM para la frecuencia
de paro por estación de trabajo.

En seguida, una segunda muestra más pequeña fue recolectada con la cantidad de paros por tipo de estación y por causa de paro. En la tabla 2 es mostrada la frecuencia y clasificación resultante de ésta segunda muestra con 189 paros.

Tabla 2. Frecuencia y clasificación de los paros por estación de la línea de ensamble.

\begin{tabular}{|c|c|c|c|c|c|}
\hline \multirow{2}{*}{$\begin{array}{c}\text { Tipo de } \\
\text { Estación }\end{array}$} & \multicolumn{5}{|c|}{ Causas de los Paros por Estación } \\
\cline { 2 - 7 } & Operador & $\begin{array}{c}\text { Herra- } \\
\text { mientas }\end{array}$ & $\begin{array}{c}\text { Materia } \\
\text { Prima }\end{array}$ & Otros & $\begin{array}{c}\text { Total Tipo } \\
\text { Estación }\end{array}$ \\
\hline A & 77 & 15 & 14 & 7 & 113 \\
\hline B & 25 & 5 & 6 & 7 & 43 \\
\hline C & 14 & 8 & 5 & 6 & 33 \\
\hline $\begin{array}{c}\text { Total } \\
\text { Causa }\end{array}$ & 116 & 28 & 25 & 20 & 189 \\
\hline
\end{tabular}

Además, las causas o motivos de los paros fueron clasificados por operador, herramientas, materia prima y otras causas (otros).

Los datos fueron agrupados de esta forma para ajustar la prueba de independencia de la cantidad de paros. Lo verificable con la prueba de independencia es encontrar si las probabilidades de que estos grupos de estaciones y de estas causas de paro ocurran simultáneamente, es igual al producto de las probabilidades individuales. Determinado lo anterior, podemos aplicar las formulas de la distribución binomial y de la multinomial.

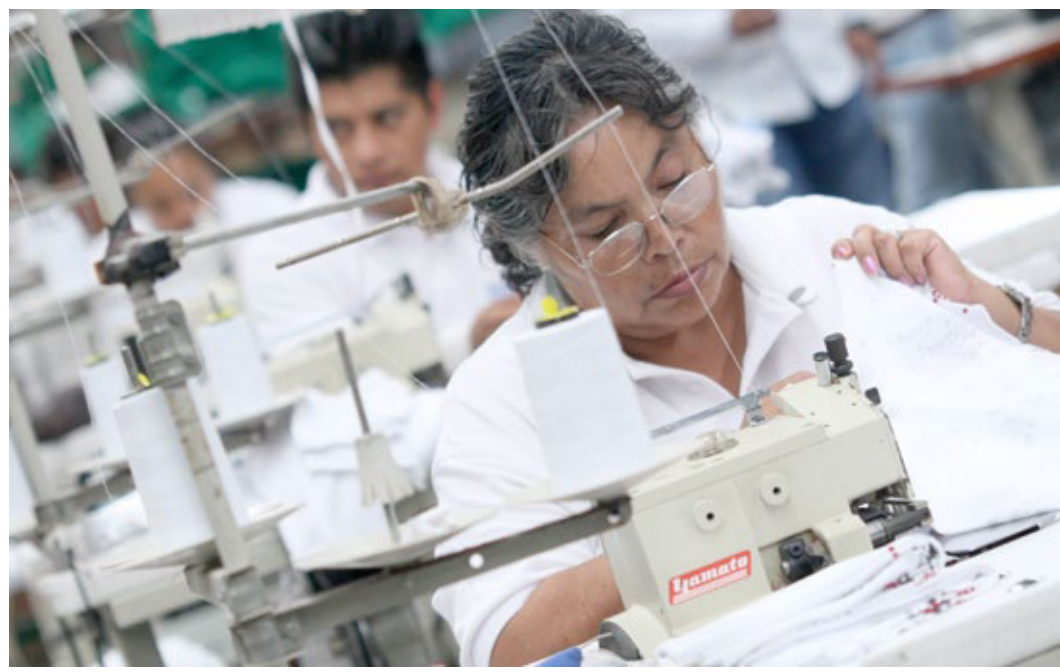




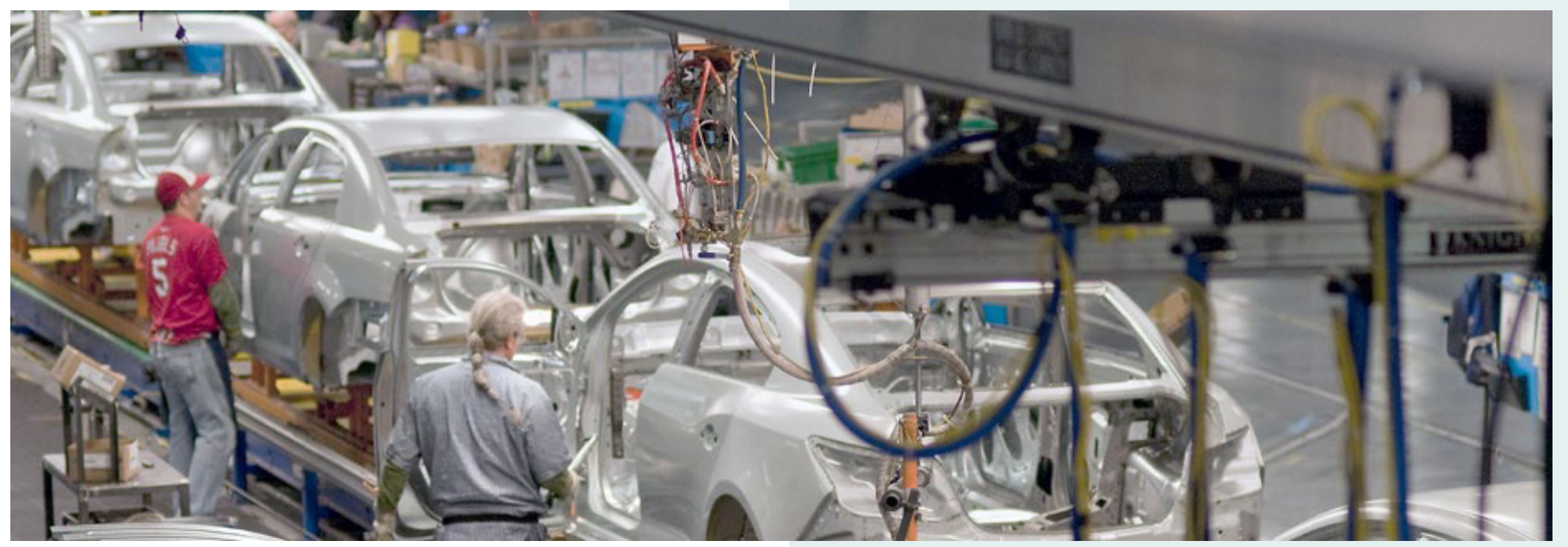

Con base en los datos de la frecuencia de paros de la línea mostrados en la tabla 2, calculamos las probabilidades para los diferentes motivos de paro y evaluamos las pruebas de hipótesis, lo que permitió verificar la credibilidad de una declaración específica para cada una de estas probabilidades. Los paros de la línea de ensamble fueron clasificados en paros debidos al operador $\left(P_{2}\right)$, herramientas, materia prima $\left(P_{3}\right)$ y a otras causas $\left(P_{4}\right)$. De los datos de la Tabla 2, con $\mathrm{p}_{\mathrm{i}}=\mathrm{x}_{\mathrm{i}} / \mathrm{n}$, con $1 \leq \mathrm{i} \leq \mathrm{k}$, fueron obtenidas las probabilidades de clase para la línea (ver Tabla 3).

Tabla 3. Probabilidades de clase a nivel línea de ensamble.

\begin{tabular}{|c|c|c|c|}
\hline$P_{1}$ & $P_{2}$ & $P_{3}$ & $P_{4}$ \\
\hline 0.61376 & 0.14815 & 0.13228 & 0.10582 \\
\hline
\end{tabular}

La credibilidad de esta sugerencia puede ser examinada con una prueba de bondad de ajuste chi-cuadrada. Por esta razón, es inferida la distribución multinomial, considerada una extensión multivariable de la distribución binomial. Cada paro era independiente y clasificado en una de las $\mathrm{k}$ clases. La prueba de independencia fue realizada para la Tabla 2 de la misma forma en que fue desarrollada por Jaime Sánchez [7], pero ahora aplicando un experimento multinomial en dos sentidos, que expresamos a continuación:

Hipótesis. $\mathrm{H}_{0}$ : El tipo de estación es independiente de la causa o motivo del paro $\mathrm{H}_{1}$ : El tipo de estación es dependiente de la causa o motivo del paro.

Estadístico de prueba:

Donde

$$
\mathrm{X}_{\mathrm{c}}^{2}=\sum_{\mathrm{i}=1}^{\mathrm{r}} \sum_{\mathrm{j}=1}^{\mathrm{c}} \mathrm{n}_{\mathrm{ij}}-\mathrm{E} \quad \mathrm{n}_{\mathrm{ij}}^{2} \quad \mathrm{E} \mathrm{n}_{\mathrm{ij}} \sim \mathrm{x}_{6}^{2}
$$

$$
\begin{gathered}
v=(\mathrm{r}-1)(\mathrm{c}-1)=(3-1)(4-1)=6 \\
\mathrm{E} \mathrm{n}_{\mathrm{ij}}=\mathrm{n}_{\mathrm{i} \cdot} \mathrm{n}_{\mathrm{j}} / \mathrm{n} \\
\text { Valor }-\mathrm{p}=\mathrm{P} \quad\left(\mathrm{x}_{\mathrm{c}}^{2}>\mathrm{x}_{6}^{2}\right)
\end{gathered}
$$

$x_{6}^{2}, 0 .{ }_{01}$ es obtenido de tal forma que $P x^{2}>x_{6}^{2}, 0.01=0.01$. Regla de decisión: $S i \mathrm{x}_{\mathrm{c}}^{2}>16.8119$ entonces rechazar $\mathrm{H}_{0}$, de otra manera no rechazar. Si el Valor- $p<\alpha$ entonces rechazar $\mathrm{H}_{0}$, de otra manera no rechazar. Obteniendo los valores de la ecuación (3) para cada $E n_{i j} y$ sustituyendo estos valores en la ecuación (1), obtenemos el valor $x_{c}^{2}=10.648$. Decisión: $x_{c}^{2}<16.8119$, entonces no rechazar $\mathrm{H}_{0}$. Además, el Valor- $\mathrm{p}=0.100 \mathrm{y} \propto=0.05$. Como el Valor- $p>\alpha$, entonces no rechazar $\mathrm{H}_{0}$. Conclusión: Los datos proveen suficiente evidencia para indicar que el tipo de estación es independiente de la causa o motivo del paro, con un nivel de significancia del $1 \%$.

Una vez comprobada la independencia, asumimos una distribución multinomial con $\mathrm{k}=4$ elementos y un conjunto de probabilidades por elemento desconocidas $p_{1}, p_{2}, p_{3}, p_{4}$. Sobre la base de un conjunto de frecuencias por elemento observado $\mathrm{x}_{1}, \mathrm{x}_{2}, \mathrm{x}_{3}, \mathrm{x}_{4}$ con $\sum_{\mathrm{i}=1}^{4} \mathrm{x}_{\mathrm{i}}=189$ , las hipótesis nulas $\mathrm{H}_{0}: \mathrm{p}_{1}=0.61376, \mathrm{p}_{2}=0.14815, \mathrm{p}_{3}=0.13228$, $\mathrm{p}_{4}=0.10582$ que establece que las probabilidades de clase toma un conjunto específico de valores, tiene un valor $p$ que puede calcularse como:

$$
\text { valor }-p=P\left\{X_{k-1}^{2} \geq X^{2}\right\}
$$

donde la prueba estadística chi-cuadrada es

$$
X^{2}=\sum_{i=1}^{3} \frac{\left(x_{i}-e_{i}\right)^{2}}{e_{i}}
$$

con frecuencias esperadas de clase o número esperado de paros de clase $i$, que es

$$
e_{i}=n \times p_{i}, \text { donde } n=189 \mathrm{y} i=1,2,3,4
$$

Sustituyendo los valores en la ecuación (6) obtuvimos: $\mathrm{X}^{2}=4.17807 \mathrm{E}-08$ con $\mathrm{k}-1=4-1=3$ grados de libertad. Por lo que la ecuación (5) es convertida en valor- $p=P\left\{x_{2}^{2} \geq 4.17807 E-08\right\}=1$. De manera alternativa, la hipótesis nula no se rechaza si $x_{k-1, \alpha}^{2} \geq X^{2}$. Al determinar el valor de $\propto=0.01$ y el de $x_{3,0.01}^{2}=11.34$ es concluido que existe suficiente evidencia estadística para no rechazar la hipótesis nula.

Así, podemos predecir que los siguientes $\mathrm{m}$ paros de la línea de ensamble tienen una distribución multinomial, y su función de masa de probabilidad conjunta es

$$
P_{\text {Linea }}\left\{X_{1}=x_{1}, \ldots, X_{4}=x_{4}\right\}=\frac{m !}{x_{1} ! \ldots x_{4} !} \times p_{1}^{x_{1}} \times \ldots \times p_{4}^{x_{4}}
$$




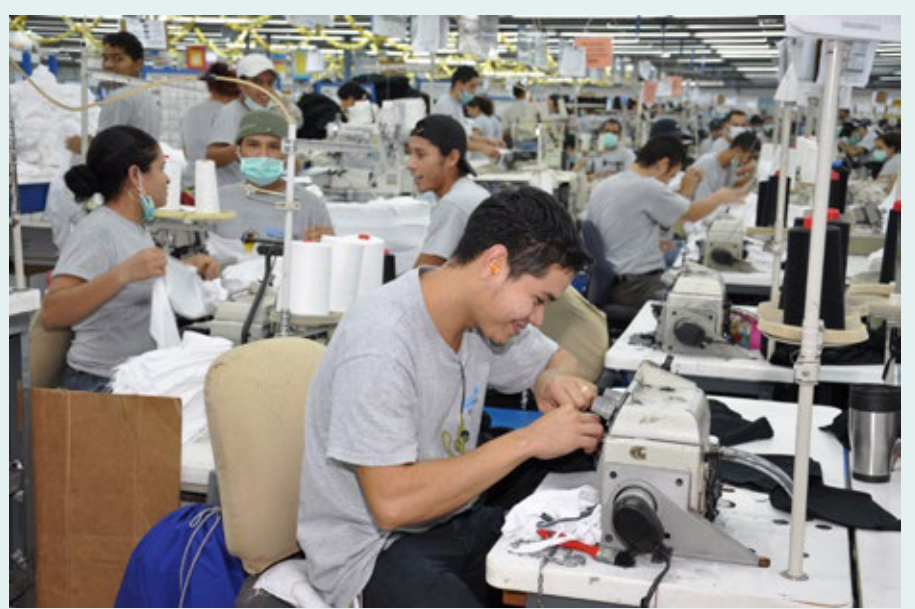

La fórmula (8) es importante tomarse en cuenta por los departamentos involucrados porque puede predecir la causa o motivo de los siguientes $m$ paros de la línea, suponiendo que un paro de línea es independiente de otro.

Posteriormente, fue realizada la prueba de bondad de ajuste chi-cuadrada a nivel de estación de trabajo, con frecuencias de clases observadas $x_{i j}$ donde los $m$ paros de línea (observaciones) fueron clasificados en una (y sólo una) de las $\mathrm{k}$ clases de las $\lambda$ estaciones de trabajo, con lo que obtuvimos:

$$
\mathrm{m}=\sum_{\mathrm{j}=1}^{\lambda} \mathrm{m}_{\mathrm{j}}=\sum_{\mathrm{i}=1}^{\mathrm{k}} \sum_{\mathrm{j}=1}^{\lambda} \mathrm{x}_{\mathrm{ij}}
$$

y las frecuencias esperadas de clase o número esperado de paros de clase ij son:

$$
e_{i j}=n_{j} \times p_{i j} \text {, donde } i=1,2,3,4 \mathrm{y} j=1,2,3
$$

Es observada una coincidencia entre las frecuencias de clase observadas y las frecuencias de clase esperadas, por lo tanto, la hipótesis nula no se rechaza para el nivel de estación de trabajo con sus respectivas probabilidades (ver tabla 4).

Tabla 4. Probabilidades de clase para la línea de ensamble a nivel estación de trabajo.

\begin{tabular}{|l|l|l|l|l|}
\hline \multirow{2}{*}{ ESTACIÓN } & \multicolumn{4}{|c|}{$\mathrm{p}_{\mathrm{ij}}$ categorías } \\
\cline { 2 - 5 } & 1 & 2 & 3 & 4 \\
\hline TIPO A & 0.68142 & 0.13274 & 0.12389 & 0.06195 \\
\hline TIPO B & 0.58140 & 0.11628 & 0.13953 & 0.16279 \\
\hline TIPO C & 0.42424 & 0.24242 & 0.15152 & 0.18182 \\
\hline
\end{tabular}

Consecuentemente, podemos predecir los próximos $\mathrm{m}_{\mathrm{i}}$ paros de la línea de ensamble a nivel de estación de trabajo a partir de la distribución multinomial, con la función de masa de probabilidad, la cual es:

$$
P_{E T_{j}}\left\{X_{1 j}=x_{1 j}, \ldots, X_{4 j}=x_{4 j}\right\}=\frac{m_{j} !}{x_{1 j} ! \ldots x_{j j} !} \times p_{1 j}^{x_{1 j}} \times \ldots \times p_{4 j}^{x_{4 j}} \text { para }=1,2,3
$$

\section{RESULTADOS}

Los datos obtenidos de la segunda muestra de la línea de ensamble fueron recolectados durante un tiempo de 8 horas, en el cual fueron recogidas 189 muestras de paros de línea. A partir de ello fue aproximada la tasa de paros de la línea de ensamble por hora como $189 / 8=23.625$ paros por hora. Una vez establecido el número de $\mathrm{m}$ paros en la línea de ensamble, con las ecuaciones (8) y (9), fueron calculados los paros y obtenida la función de masa de probabilidades conjunta a nivel de línea. En la Tabla 5, es presentada la asignación de paros para diferentes valores de $m$ y la función de masa de probabilidades para la línea de ensamble a nivel de línea.

\begin{tabular}{|c|c|c|c|c|c|c|c|c|c|c|c|}
\hline m paros & $x_{1}$ & $X_{2}$ & $x_{3}$ & $X_{4}$ & $\mathbf{P}_{\text {Linea }}$ & m paros & $x_{1}$ & $X_{2}$ & $X_{3}$ & $X_{4}$ & $\mathbf{P}_{\text {Linea }}$ \\
\hline 1 & 1 & 0 & 0 & 0 & 0.61376 & 13 & 8 & 2 & 2 & 1 & 0.03159 \\
\hline 2 & 2 & 0 & 0 & 0 & 0.37670 & 14 & 9 & 2 & 2 & 1 & 0.03016 \\
\hline 3 & 2 & 1 & 0 & 0 & 0.16742 & 15 & 9 & 2 & 2 & 2 & 0.02394 \\
\hline 4 & 2 & 1 & 1 & 0 & 0.08858 & 16 & 10 & 2 & 2 & 2 & 0.02351 \\
\hline 5 & 3 & 1 & 1 & 0 & 0.09061 & 17 & 10 & 3 & 2 & 2 & 0.01974 \\
\hline 6 & 4 & 1 & 1 & 0 & 0.08342 & 18 & 11 & 3 & 2 & 2 & 0.01982 \\
\hline 7 & 4 & 1 & 1 & 1 & 0.06179 & 19 & 12 & 3 & 2 & 2 & 0.01926 \\
\hline 8 & 5 & 1 & 1 & 1 & 0.06068 & 20 & 12 & 3 & 3 & 2 & 0.01699 \\
\hline 9 & 6 & 1 & 1 & 1 & 0.05587 & 21 & 13 & 3 & 3 & 2 & 0.01684 \\
\hline 10 & 6 & 2 & 1 & 1 & 0.04138 & 22 & 14 & 3 & 3 & 2 & 0.01624 \\
\hline 11 & 7 & 2 & 1 & 1 & 0.03991 & 23 & 14 & 3 & 3 & 3 & 0.01318 \\
\hline 12 & 7 & 2 & 2 & 1 & 0.03168 & & & & & & \\
\hline
\end{tabular}

Tabla 5. Localización de los paros para los diferentes $\mathrm{m}$ paros y su función de masa de probabilidad a nivel línea. 
Es importante observar el número actual de $\mathrm{m}$ paros hasta el primer paro de $x_{\mathrm{i}}$ El primer paro debido al operador $x_{1}$ es probable que aparezca cuando $m=1$, el primer paro debido a la herramienta $x_{2}$ es probable que aparezca cuando $m=3$, el primer paro debido a materias primas $x_{3}$ es probable que aparezca cuando $m=4$ y el primer paro debido a otras causas $\mathrm{X}_{4}$ es probable que aparezca cuando $m=7$.

También fue observada en la función de masa de probabilidad (8) que si $\mathrm{x}_{1}=1$ y $\mathrm{x}_{2}=\mathrm{x}_{3}=\mathrm{x}_{4}=0$, siendo un caso especial donde en la línea de ensamble es presentado sólo un paro debido a la primera clase y ningún paro debido a las otras clases, la probabilidad puede obtenerse de la ecuación (8) como, $P_{\text {Linea }}\left\{X_{1}=1, X_{2}=0, X_{3}=0, X_{3}=0\right\}=\frac{1 !}{1: 0 ! 0 ! 0 !} \times p_{1}^{1} \times p_{2}^{0} \times p_{3}^{0} \times p_{4}^{0}=p_{1}=0.61376$.

Después, si $x_{1}=2 y, x_{2}=x_{3}=x_{4}=0$ en la línea de ensamble es presentado sólo dos paros debido a la primera clase, obtenemos que $\left(p_{1}\right)^{2}=0.37670$. Lo mismo sucede si sólo es presentado un paro debido a la segunda clase $P_{\text {Linea }}\left\{X_{2}=1\right.$ y $\left.X_{1}=X_{3}=X_{4}=0\right\}=p_{2}=0.14815$ en la línea de ensamble, etc.

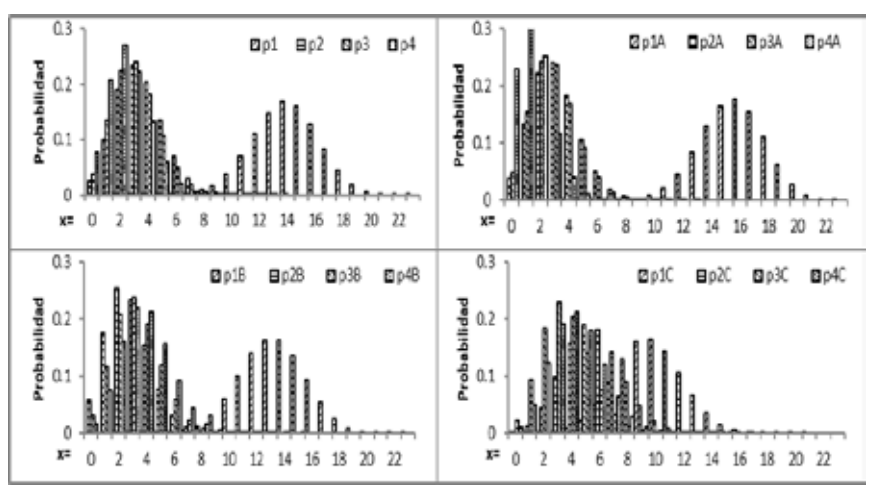

Figura 2. Gráficas de probabilidad por causa de paro con respecto al número de paros ( $\mathrm{x}=23$ paros por hora) para la línea de ensamble y para los tipos de estaciones.

Además, al investigar cómo las probabilidades de las diferentes categorías de $p_{i}$ y $p_{i j}$ afectan, podrá ser mejorada la eficiencia de la línea de ensamble. Así, fue realizado un estudio numérico asumiendo la distribución binomial por ser la más adecuada en el caso (a) $\mathrm{X}_{\mathrm{i}} \approx \mathrm{B}\left(23, \mathrm{p}_{\mathrm{i}}\right)$, con $\mathrm{i}=1,2,3,4$ para el nivel línea y el caso $(b) X_{i j} \approx B\left(23, p_{i j}\right)$, con $i=1,2,3,4$ y $\mathrm{j}=1,2,3$ para el nivel de estaciones de trabajo. En ambos casos, la variable aleatoria de interés fue el número de paros obtenidos dentro de un número fijo de 23 paros totales independientes, cada uno de los cuales tiene una probabilidad constante $p_{i}$ y $p_{i j}$ de paros para el caso (a) y para el caso (b), respectivamente (ver figura 2 ).

De la figura 2, son hechas las siguientes observaciones: Toda la línea de ensamble muestra una marcada presencia de paros debidos al operador cuando $x>8$ y cuando $x<8$ muestra presencia de paros debido a las herramientas, materias primas y otras causas.

Conclusiones
Es más probable que estén presentes los paros de línea a causa de los operadores debido a que representan el $61.37 \%$ de todos los paros, por lo que se requerirá maximizar las habilidades y conocimientos de los operadores sobre las operaciones a realizar en las estaciones de trabajo, además de maximizar las habilidades y conocimientos sobre las características y condiciones de las materias primas a ensamblar y sobre las herramientas a utilizar, con una formación adecuada de acuerdo a las necesidades específicas de cada estación de trabajo y de cada individuo.

La atención de problemas en la línea de ensamble exige un equipo de individuos con conciencia, en donde los operadores de la línea de ensamble son quienes tienen la capacidad de descubrir, corregir anomalías y aprender de esos errores para no continuar con ellos. Además, es necesario buscar que los operadores cuenten con las condiciones óptimas con el fin de aumentar la efectividad de la operación de la línea.

\section{BIBLIOGRAFÍA}

[1] A. Simboli, R. Taddeo and A. Morgante, "Value and Wastes in Manufacturing. An Overview and a New Perspective Based on Eco-Efficiency", Administrative Sciences, vol. 4, no 3, p. 173-191, 4 July 2014.

[2] S. Shingo, A Study of the Toyota Production System. From an Industrial Engineering Viewpoint, Cambridge, MA: Productivity Press, 1989.

[3] B. W. Niebel y A. Freivalds, Ingeniería Industrial. Métodos, estándares y diseño del trabajo, Duodécima ed., México, D.F.: McGraw Hill, 2009.

[4] M. L. Pinedo, Planning and Scheduling in Manufacturing and Services, Second edition ed., New York, NY: Springer Science+Business Media, LLC, 2009.

[5] X. Gu, S. Lee, X. Liang, M. Garcellano, M. Diederichs and J. $\mathrm{Ni}$, "Hidden maintenance opportunities in discrete and complex production lines", Expert Systems with Applications, vol. 40, no 11, p. 4353-4361, 1 September 2013.

[6] P. H. Tsarouhas, "Classification and calculation of primary failure modes in bread productionline", Reliability Engineering and System Safety, Vol 94., p. 551-557, 2009.

[7] J. Sánchez Leal, Estadística, Primera ed., Ciudad Juárez, Chihuahua: Taller Editorial del Instituto Tecnológico de Ciudad Juárez, 2001.

[8] A. Hayter, Probability and Statistics for Engineers and Scientists, Fourth ed., Boston, Massachusetts: Cengage, 2012.

[9] P. Pallmann y L. A. Hothorn, "Analysis of means: a generalized approach using R", Journal of Applied Statistics, vol. 43, n 8, p. 1541-1560, 2016.

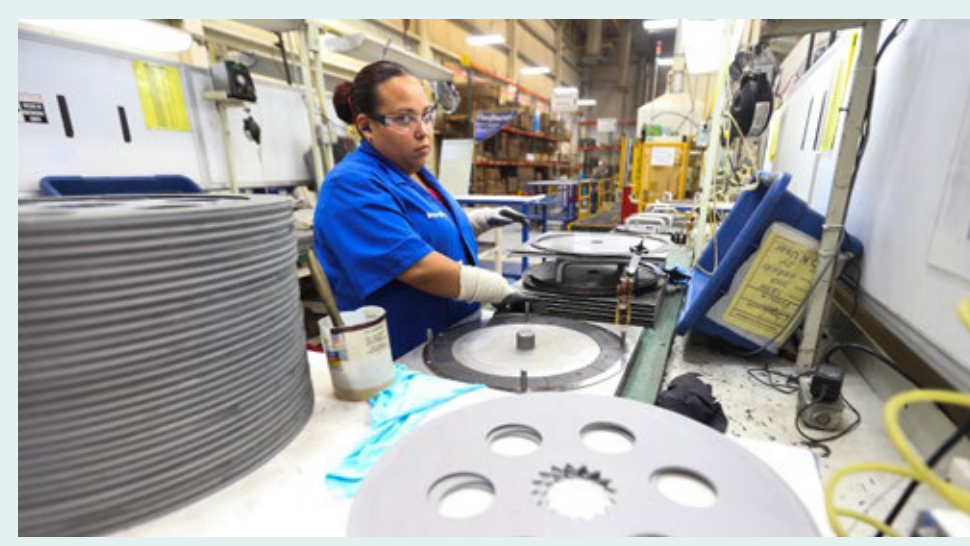

